\title{
Thermal Transport in Radiative Nanofluids by Considering the Influence of Convective Heat Condition
}

\author{
Adnan $\mathbb{D}^{1},{ }^{1}$ Waqas Ashraf, ${ }^{2}$ Abdulaziz H. Alghtani, ${ }^{3}$ Ilyas Khan $\mathbb{D},{ }^{4}$ and M. Andualem $\mathbb{D}^{5}$ \\ ${ }^{1}$ Department of Mathematics, Mohi-ud-Din Islamic University, Nerian Sharif, AJ\&K 12080, Pakistan \\ ${ }^{2}$ Department of Applied Mathematics and Statistics (AM\&S), Institute of Space Technology (IST), Islamabad 44000, Pakistan \\ ${ }^{3}$ Mechanical Engineering Department at Taif University, P.O. Box 11099, Taif 21944, Saudi Arabia \\ ${ }^{4}$ Department of Mathematics, College of Science Al-Zulfi, Majmaah University, Al-Majmaah 11952, Saudi Arabia \\ ${ }^{5}$ Department of Mathematics, Bonga University, Bonga, Ethiopia
}

Correspondence should be addressed to Adnan; adnan_abbasi89@yahoo.com and M. Andualem; mulugetaandualem4@gmail.com

Received 18 October 2021; Revised 27 November 2021; Accepted 13 December 2021; Published 5 January 2022

Academic Editor: Taza Gul

Copyright (c) 2022 Adnan et al. This is an open access article distributed under the Creative Commons Attribution License, which permits unrestricted use, distribution, and reproduction in any medium, provided the original work is properly cited.

\begin{abstract}
The analysis of nanofluid dynamics in a bounded domain attained much attention of the researchers, engineers, and industrialists. These fluids became much popular in the researcher's community due to their broad uses regarding the heat transfer in various industries and fluid flowing in engine and in aerodynamics as well. Therefore, the analysis of Cu-kerosene oil and $\mathrm{Cu}$-water is organized between two Riga plates with the novel effects of thermal radiations and surface convection. The problem reduced in the form of dimensionless system and then solved by employing variational iteration and variation of parameter methods. For the sake of validity, the results checked with numerical scheme and found to be excellent. Further, it is examined that the nanofluids move slowly by strengthen $\mathrm{Cu}$ fraction factor. The temperature of $\mathrm{Cu}$-kerosene oil and $\mathrm{Cu}$-water significantly rises due to inducing thermal radiations and surface convection. The behaviour of shear stresses is in reverse proportion with the primitive parameters, and local Nusselt number increases due to varying thermal radiations, Biot number, and fraction factor, respectively.
\end{abstract}

\section{Introduction}

The heat transfer caused troubles for industrialists and engineers because reasonable amount of it required to cope many industrial and engineering processes. Therefore, a second generation of the fluid was developed called nanofluids [1]. These fluids are the composition of two or more than two nanoscaled particles in the host liquid. Due to reasonable thermal performance of these fluids, researchers focused on the study of nanofluids and its advancements. The applications of these fluids fall in the field medical sciences, electronic devices, paint industries, drug delivery system, the study of bionanofluids in human veins and arteries, the reaction of nanomaterials by taking blood as a host fluid, coating of various aircraft parts, and in many other potential fields of interest. Therefore, it is imperative to address the comparative heat transfer analysis in different nanofluids.
To improve the heat transport in nanofluids, many theoretical models have been suggested; some of them are Hamilton and Crossers model, Maxwell Garnett model, KKL model, Buongiorno's model, etc. By utilizing aforementioned thermal conductivity models, many researchers explored the problems of nanofluids in various geometries and studied their dynamics under various conditions. In 2016, Mohyud-Din et al. [2] discussed the mass and heat transfer analysis in opening (diverging) and narrowing (converging) channel. They assumed that walls of the channel capable to stretching and shrinking. The investigation of heat transfer over a stretchable surface by inducing an MHD and thermal radiation examined by Khan et al. [3] in 2016.

Sheikholeslami [4] explored the behaviour of nanofluid under the governing parameters and Lorentz forces. The effect of Lorentz forces on the nanoliquid past a porous 
cylinder with the help of Darcy model was reported in [5]. Adnan et al. [6] explored the second-grade fluid problem between opening/narrowing walls by inducing cross diffusion effects in the constitutive model. Also, they demonstrated the effects of other flow parameters ingrained in the flow model on the velocity, temperature, and concentration fields. The analysis of two phase nanoliquid model by plugging the effects of Lorentz forces is presented in [7]. Another imperative inspection of the nanoliquid dynamics is reported in [8]. For thermal enhancement, they induced KKL correlation in the model.

Influence of thermal radiation between oblique walls was reported by Adnan et al. [9]. They assumed that the boundaries of the channel are capable to stretching and shrinking. Furthermore, they solved the model and explored the effects of various flow parameters in the flow field. The significant analysis regarding the heat transfer in nanoliquids under multiple flow scenarios is reported in $[10,11]$. The dynamics of radiative nanoliquid by considering combined convection is reported by Gul et al. [12]. The important analysis of the nanoliquids under imposed magnetic field and other governing quantities is conducted in $[13,14]$, respectively.

The analysis of the squeezing flow has great significance in the light of its applications in different industries. These applications comprised in the field of biomechanics, mechanical engineering, chemical engineering, and in the food industries. The earlier work in this era was done by Stefan [15]. The behaviour of squeezed fluid was investigated by Rashidi et al. [16]. Another analysis related to squeezed flow is done by Khan et al. [17]. Gailitis and Lielausis [18] presented the study of fluid flowing between Riga plates. The model is solved via suitable technique and then explained the physical results deeply.

The dynamics of bioconvection nanoliquid regarding the heat transfer are examined in [19]. They organized the study in $3 \mathrm{D}$, and the problem is developed by Buongiorno nanoliquid model and then found the solution analytically. In 2019, Shamshuddin et al. [20] reported the novel influences of ohmic heating and magnetic field in the flow of nanoliquid between two Riga sheets. They analyzed the model analytically and provided pictorial results against the pertinent flow quantities. The investigation of local heat transport mechanism in the nanoliquid and transportation of the shears stresses at the walls are also reported in their study. The effect of partial slip in the nanoliquid flow over a spinning geometry is reported in [21]. They used the nanoliquid composed by $\mathrm{MgO}$ and discussed the heat transport properties. Another imperative heat transfer treatment in bioconvection nanoliquid under the influences of Lorentz forces and internal heat source is explored in [22]. Some significant investigations related to nanoliquids under multiple conditions are reported in [23-25].

Thermal diffusion and heat generation are very important physical phenomena that play significant role in the nanofluid heat transport performance. In this regard, a potential study is reported in [26] past through porous oscillating plate placed in vertical plane. The authors computed the results and for the shear stresses, Nusselt number and furnished graphical results for the velocity and temperature distribution. Another potential work for the heat transfer in the nanofluid under the effects of imposed Lorentz forces is discussed in [27]. Thin film flows attained much attention of the industrialists due to their broad applications in many industries. The analysis of heat transport in the nanofluids over a thin film is very prominent. Therefore, a recent study in this regard is reported in [28]. Theoretical study of the flow models under varying flow conditions has its own importance, and researchers paved their attentions to analyze the dynamics of fluids through theoretical approaches. In this regard, a study is reported in [29] by considering the flow inside the cavity filled with nanoliquid. The authors treated the model numerically and authenticate their results with previously published work. The study of different flow parameters and their impacts on the heat transfer is organized in [30]. It is reported that the temperature drops due higher thermophoresis effects.

From the available science literature, it is noted that the analytical heat transport mechanism in water and kerosene oil saturated by $\mathrm{Cu}$ nanomaterials is not investigated so far. Therefore, the analysis is organized to examine the nanofluid characteristics between Riga plates. The model is obtained via similarity transforms, and then for solution purpose, two different analytical techniques (VIM and VPM) are implemented. The solutions are tabulated successfully and discussed the graphical results comprehensively within desired domain.

\section{Modelling of the Flow Configuration}

In this study, we consider the squeezed flow of copper nanofluids between Riga plates. The plates are situated at $y=0$ (lower plate) and $y=h(t)=\left(a / v_{f}(1-\gamma t)\right)^{-1 / 2}$ (upper plate). The lower Riga plate is capable to stretching with velocity $U_{w}=a x(1-\gamma t)^{-1}$. The base liquids diluted with $\mathrm{Cu}$ nanoparticles flow with velocity $v_{h}=d h / d t$ between Riga plates. Furthermore, thermal radiation effects are under consideration. Figure 1 demonstrates flow scenario for the nanofluids between Riga plates.

The squeezing flow of nanofluids past a Riga plate described by the following system [31]:

$$
\begin{gathered}
\frac{\partial u}{\partial x}+\frac{\partial v}{\partial y}=0 \\
\frac{\partial u}{\partial t}+u \frac{\partial u}{\partial x}+v \frac{\partial u}{\partial y}=\frac{1}{\rho_{n f}}\left[-\frac{\partial p}{\partial x}+\mu_{n f}\left(\frac{\partial^{2} u}{\partial x^{2}}+\frac{\partial^{2} u}{\partial y^{2}}\right)\right]+\frac{\pi j_{0} M_{0} e^{(-\pi y / b)}}{8 \rho_{n f}} \\
\frac{\partial v}{\partial t}+u \frac{\partial v}{\partial x}+v \frac{\partial v}{\partial y}=\frac{1}{\rho_{n f}}\left[-\frac{\partial p}{\partial y}+\mu_{n f}\left(\frac{\partial^{2} v}{\partial x^{2}}+\frac{\partial^{2} v}{\partial y^{2}}\right)\right] \\
\frac{\partial T}{\partial t}+u \frac{\partial T}{\partial x}+v \frac{\partial T}{\partial y}=\frac{k_{n f}}{\left(\rho C_{p}\right)_{n f}}\left[\frac{\partial^{2} T}{\partial x^{2}}+\frac{\partial^{2} T}{\partial y^{2}}\right]-\frac{1}{\left(\rho C_{p}\right)_{n f}} \frac{\partial}{\partial y}\left(q_{r}\right)
\end{gathered}
$$

Equation (1) is the mathematical expression for 


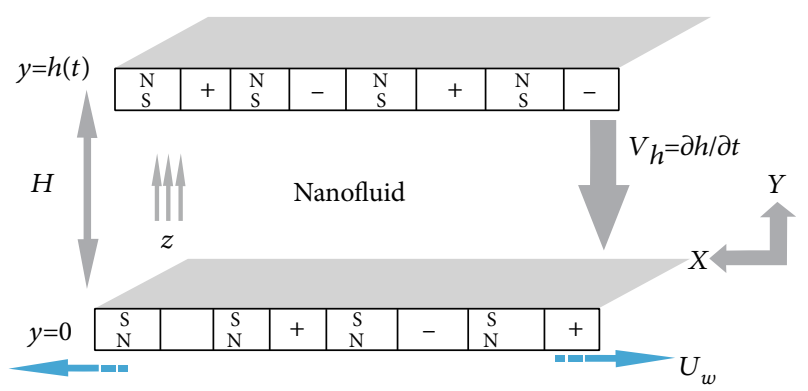

Figure 1: The flow scenario between Riga plates.

conservation of mass. Equations (2) and (3) presenting the $x$ and $y$ components of the momentum equation and energy equation are described by Equation (4). The term $q_{r}$ comprised in energy equation is defined as:

$$
q_{r}=-\frac{4 \sigma^{*}}{3 k^{*}} \frac{\partial}{\partial y}\left(T^{4}\right)
$$

Here, $\sigma^{*}$ denotes the Stefan-Boltzmann constant, and $q_{r}$ denotes the radiative heat flux. By expanding $T^{4}$ in wellknown Taylor series centered at $T_{h}$, we arrived with the following form:

$$
T^{4}=\sim 4 T_{h}^{3}-3 T_{h}^{4}
$$

By utilizing above expressions, updated version of energy equation is as follows:

$\frac{\partial T}{\partial t}+u \frac{\partial T}{\partial x}+v \frac{\partial T}{\partial y}=\frac{k_{n f}}{\left(\rho C_{p}\right)_{n f}}\left[\frac{\partial^{2} T}{\partial x^{2}}+\frac{\partial^{2} T}{\partial y^{2}}\right]-\frac{1}{\left(\rho C_{p}\right)_{n f}} \frac{16 \sigma^{*}}{3 k^{*}} T_{h}^{3} \frac{\partial^{2} T}{\partial y^{2}}$.

For under consideration flow model of nanofluid, the flow conditions are as follows [31]:

$$
\begin{gathered}
u=U_{w}(x)=(1-\gamma t)^{-1} a x \\
v=0,-\frac{k \partial T}{\partial y}=\frac{k_{1}}{\left(T_{f}-T\right)^{-1}}
\end{gathered}
$$

$$
\mathrm{u}=0, v=\frac{d h}{d t}=-\frac{\gamma}{2}\left(\frac{a(1-\gamma t)}{v_{f}}\right)^{-1 / 2}, T=T_{h}
$$

In Equations (8) and (10), $\rho_{n f}$ represents the nanofluid density, $\mu_{n f}$ represents the effective dynamic viscosity, and $\left(\rho C_{p}\right)_{n f}$ represents the effective heat capacity. Here, we used Brinkman and Maxewell Garnett model for effective dynamic viscosity and thermal for the thermal conductivity of the nanofluid [32]:

$$
\begin{gathered}
\rho_{n f}=\frac{(1-\phi)}{\rho_{f}^{-1}}+\frac{\phi}{\rho_{s}^{-1}}, \\
\mu_{n f}=\frac{\mu_{f}}{(1-\phi)^{2.5}}, \\
\left(\rho C_{p}\right)_{n f}=\frac{(1-\phi)}{\left(\rho C_{p}\right)_{f}^{-1}}+\frac{\phi}{\left(\rho C_{p}\right)_{s}^{-1}}, \\
k_{n f}=k_{f}\left[\frac{k_{s}+2 k_{f}-2 \phi\left(k_{f}-k_{s}\right)}{k_{s}+2 k_{f}+\phi\left(k_{f}-k_{s}\right)}\right] .
\end{gathered}
$$

In above expressions, $\phi$ is called the nanoparticle volume fraction.

The dimensionless feasible transformations for the flow model are given by the following expressions:

$$
\begin{gathered}
\psi=\left(\frac{a v_{f}}{1-\gamma t}\right)^{1 / 2} x F(\eta), \eta=\frac{y}{h(t)}, \\
u=U_{w} F^{\prime}(\eta), \\
v=-\left(\frac{a v_{f}}{1-\gamma t}\right)^{1 / 2}, \\
\theta(\eta)=\left(\frac{T-T_{f}}{T_{h}-T_{f}}\right) .
\end{gathered}
$$

Applying these transformations and suitable differentiation in Equations (2)-(7), the following system is attained:

$$
\begin{gathered}
F^{\prime \prime \prime \prime}(\eta)+\left[(1-\phi)+\phi\left\{\frac{\rho_{s}}{\rho_{f}}\right\}\right](1-\phi)^{2.5}\left[-F^{\prime}(\eta) F^{\prime \prime}(\eta)+F(\eta) F^{\prime \prime \prime}(\eta)-\frac{\beta}{2}\left(3 F^{\prime \prime}(\eta)+\eta F^{\prime \prime \prime}(\eta)\right)\right]-(1-\phi)^{2.5} Z^{*} B^{*} \operatorname{Exp}\left(-B^{*} \eta\right)==0 \\
{\left[1+R d\left[\frac{k_{s}+2 k_{f}-2 \phi\left(k_{f}-k_{s}\right)}{k_{s}+2 k_{f}+\phi\left(k_{f}-k_{s}\right)}\right]^{-1}\right] \theta^{\prime \prime}(\eta)+\left[\frac{k_{s}+2 k_{f}-2 \phi\left(k_{f}-k_{s}\right)}{k_{s}+2 k_{f}+\phi\left(k_{f}-k_{s}\right)}\right]^{-1}\left[\left\{(1-\phi)+\phi\left\{\frac{\left(\rho C_{p}\right)_{s}}{\left(\rho C_{p}\right)_{f}}\right\}\right\} \operatorname{Pr}\left\{F(\eta) \theta^{\prime}(\eta)-\frac{\beta \eta}{2} \theta^{\prime}(\eta)\right\}\right]=0 .}
\end{gathered}
$$


Supporting boundary conditions for above flow model in dimensionless form is given in Equations (14) and (15) for upper and lower Riga plates:

$$
\begin{gathered}
F^{\prime}(\eta=0)=1, F(\eta=1)=\frac{\beta}{2}, F^{\prime}(\eta=1)=0, F(\eta=0)=0 \\
\theta(\eta=1)=0, \theta^{\prime}(\eta=0)=-B_{i}(1-\theta(\eta=0)) .
\end{gathered}
$$

Also, squeeze number $\beta$, Prandtl number $\mathrm{Pr}$, and radiation parameter $\mathrm{Rd}$ are described by the following expressions:

$\beta=\frac{\gamma}{a}, \quad \operatorname{Pr}=\frac{\mu_{f}\left(C_{p}\right)_{f}}{k_{f}}, R d=\frac{16 \sigma^{*} T_{h}^{3}}{3 k^{*} k_{f}}, B_{i}=-\frac{h_{1}}{k} \sqrt{\nu_{f}(1-\gamma t) / a}$.

The dimensional expressions for local heat transport rate and walls shear stresses are

$$
\begin{gathered}
C_{F}=\frac{\tau_{w}}{\rho_{n f} U_{w}^{2}}, \\
N u_{x}=\frac{x q_{w}}{k_{f}\left(T-T_{h}\right)}+q_{r} .
\end{gathered}
$$
$\downarrow_{y=h(t)}$

Here, $\quad \tau_{w}=\mu_{n f}(\partial u / \partial y) \downarrow_{y=h(t)} \quad$ and $\quad q_{w}=-k_{n f}(\partial T / \partial y)$

Finally got the following version:

$$
\begin{aligned}
& C_{F}^{\nu} \sqrt{\left(\operatorname{Re}_{x}\right)}=\frac{1}{A_{1}(1-\phi)^{2.5}} F^{\prime \prime}(\eta=1) . \\
& N u_{x} \sqrt{\frac{(1-\gamma t)}{\left(\operatorname{Re}_{x}\right)}}=-\left(A_{3}+\mathrm{Rd}\right) \theta^{\prime}(\eta=1) .
\end{aligned}
$$

where $\mathrm{Re}_{x}$ is called the local Reynolds number and is $\mathrm{Re}_{x}$ $=x U_{w} / \nu_{f}$. Furthermore, $A_{1}$ and $A_{3}$ are as follows:

$$
\begin{gathered}
A_{1}=(1-\phi)+\frac{\phi}{\left(\rho_{s} / \rho_{f}\right)^{-1}}, \\
A_{3}=\left[\frac{\left(k_{s}+2 k_{f}\right)-2 \phi\left(k_{f}-k_{s}\right)}{\left(k_{s}+2 k_{f}\right)+\phi\left(k_{f}-k_{s}\right)}\right] .
\end{gathered}
$$

\section{Solution of the Model}

In this section, solutions for the model are performed. For said purpose, we employed analytical and numerical methods. First subsection deals with the study of solutions found by variation of parameters method, and the second one deals with the study of solutions found by variational iteration method. Numerical solution and error analysis are also tabulated.
3.1. Solution Using VPM. The solution for the model by utilizing VPM is performed in this subsection. The procedure is adopted by the following steps:

$$
\begin{aligned}
F_{n+1}(\eta)= & F(0)+\eta F^{\prime}(0)+\frac{\eta^{2}}{2 !} F^{\prime \prime}(0)+\frac{\eta^{3}}{3 !} F^{\prime \prime \prime}(0) \\
& -\int_{0}^{\eta} \frac{(s \eta-s+(-\eta s+\eta))^{3}}{3 !}\left[\left[(1-\phi)+\phi\left\{\frac{\rho_{s}}{\rho_{f}}\right\}\right](1-\phi)^{2.5}\right. \\
& \cdot\left[F_{n}^{\prime}(s) F_{n}^{\prime}(s)-F_{n}(s) F_{n}^{\prime}(s)-\frac{\beta}{2}\left(3 F_{n}^{\prime}(s)+s F_{n}^{\prime}(s)\right)\right] \\
& \left.-(1-\phi)^{2.5} Z^{*} B^{*} \operatorname{Exp}\left(-B^{*} \eta\right)\right] d s, n \geq 0, \\
\theta_{n+1}(\eta)= & \theta(0)+\eta \theta^{\prime}(0)-\frac{1}{\left(1+\operatorname{Rd} / A_{3}\right)} \int_{0}^{\eta} \frac{\left(\eta^{2} s-s+\left(\eta-s \eta^{2}\right)\right)}{1 !} \\
& \cdot\left[\frac{A^{*}}{A_{3}} \operatorname{Pr}\left\{F_{n}(s) \theta_{n}^{\prime}(s)-\frac{\beta s}{2} \theta_{n}^{\prime}(s)\right\}\right] d s, n \geq 0,
\end{aligned}
$$

where $A^{*}=\left\{(1-\phi)+\phi\left\{\left(\rho C_{p}\right)_{s} /\left(\rho C_{p}\right)_{f}\right\}\right\}$

By using the conditions $(\eta=0)$ and putting $F^{\prime \prime}(0)=\alpha_{1}^{*}$ , $F^{\prime \prime \prime}(0)=\alpha_{2}^{*}$, and $\theta(0)=\alpha_{3}^{*}$, above recursive relation takes the following form:

$$
\begin{aligned}
F_{n+1}(\eta)= & \eta+\frac{\eta^{2}}{2 !} \alpha_{1}^{*}+\frac{\eta^{3}}{3 !} \alpha_{2}^{*} \\
& \left.-\int_{0}^{\eta} \frac{(s \eta-s+(-\eta s+\eta))^{3}}{3 !}\right]\left[(1-\phi)+\phi\left\{\frac{\rho_{s}}{\rho_{f}}\right\}\right](1-\phi)^{2.5} \\
& \left.\cdot\left[F_{n}^{\prime}(s) F_{n}^{\prime}(s)-F_{n}(s) F_{n}^{\prime}(s)-\frac{\beta}{2}\left(3 F_{n}^{\prime}(s)+s F_{n}^{\prime}(s)\right)\right]\right] \\
- & (1-\phi)^{2.5} Z^{*} B^{*} \operatorname{Exp}\left(-B^{*} \eta\right) d s, n \geq 0, \\
\theta_{n+1}(\eta)= & \alpha_{3}^{*}+\eta\left(-B_{i}(1-\theta(0))\right) \\
& -\frac{1}{\left(1+\operatorname{Rd} / A_{3}\right)} \int_{0}^{\eta} \frac{\left(\eta^{2} s-s+\left(\eta-s \eta^{2}\right)\right)}{1 !} \\
& \cdot\left[\frac{A^{*}}{A_{3}} \operatorname{Pr}\left\{F_{n}(s) \theta_{n}^{\prime}(s)-\frac{\beta s}{2} \theta_{n}^{\prime}(s)\right\}\right] d s, n \geq 0 .
\end{aligned}
$$

In more comprehensive form, Equations (21) and (22) become:

$$
\begin{aligned}
F_{n+1}(\eta)= & F_{0}(\eta)-\int_{0}^{\eta} \frac{(s \eta-s+(-\eta s+\eta))^{3}}{3 !} \\
& \cdot\left[\left[(1-\phi)+\phi\left\{\frac{\rho_{s}}{\rho_{f}}\right\}\right](1-\phi)^{2.5}\right. \\
& \left.\cdot\left[F_{n}^{\prime}(s) F_{n}^{\prime}(s)-F_{n}(s) F_{n}^{\prime}(s)-\frac{\beta}{2}\left(3 F_{n}^{\prime}(s)+s F_{n}^{\prime}(s)\right)\right]\right] \\
& -(1-\phi)^{2.5} Z^{*} B^{*} \operatorname{Exp}\left(-B^{*} \eta\right) d s, n \geq 0,
\end{aligned}
$$




$$
\begin{aligned}
\theta_{n+1}(\eta)= & \theta_{0}(\eta)-\frac{1}{\left(1+\mathrm{Rd} / A_{3}\right)} \int_{0}^{\eta} \frac{\left(\eta^{2} s-s+\left(\eta-s \eta^{2}\right)\right)}{1 !} \\
& \cdot\left[\frac{A^{*}}{A_{3}} \operatorname{Pr}\left\{F_{n}(s) \theta_{n}^{\prime}(s)-\frac{\beta s}{2} \theta_{n}^{\prime}(s)\right\}\right] d s, n \geq 0 .
\end{aligned}
$$

Here,

$$
\begin{gathered}
F_{0}(\eta)=\eta+\frac{\eta^{2}}{2 !} \alpha_{1}^{*}+\frac{\eta^{3}}{3 !} \alpha_{2}^{*}, \\
\theta_{0}(\eta)=\alpha_{3}^{*}+\eta\left(-B_{i}(1-\theta(0))\right),
\end{gathered}
$$

and $\alpha_{1}^{*}, \alpha_{2}^{*}$, and $\alpha_{3}^{*}$ are unknown constant and can be determined by utilizing remaining boundary conditions. Other approximations of the solution can be calculated for varying $n$.

3.2. Solution Using VIM. The solution procedure by using VIM is followed as follows:

$$
\begin{aligned}
F_{n+1}(\eta)= & F_{n}(\eta)+\int_{0}^{\eta}(-1)^{4} \frac{(s \eta+s+(-\eta s-\eta))^{3}}{3 !} \\
& \cdot\left[F_{n}^{\prime \prime}(s)+\left[(1-\phi)+\phi\left\{\frac{\rho_{s}}{\rho_{f}}\right\}\right](1-\phi)^{2.5}\right. \\
& \left.\cdot\left[F_{n}^{\prime}(s) F_{n}^{\prime}(s)-F_{n}(s) F_{n}^{\prime \prime}(s)-\frac{\beta}{2}\left(3 F_{n}^{\prime}(s)+s F_{n}^{\prime}(s)\right)\right]\right] \\
& -(1-\phi)^{2.5} Z^{*} B^{*} \operatorname{Exp}\left(-B^{*} \eta\right) d s, n \geq 0, \\
\theta_{n+1}(\eta)=\theta_{n}(\eta)+\int_{0}^{\eta} \frac{\left(\eta^{2} s+s+\left(-\eta-s \eta^{2}\right)\right)}{1 !} & \cdot\left[\left(1+\frac{\mathrm{Rd}}{A_{3}}\right) \theta_{n}^{\prime}(s)+\frac{A^{*}}{A_{3}} \operatorname{Pr}\left\{F_{n}(s) \theta_{n}^{\prime}(s)-\frac{\beta s}{2} \theta_{n}^{\prime}(s)\right\}\right] d s, n \geq 0 .
\end{aligned}
$$

In order to initiate the recursive relation for variational iteration method, we need initial guesses $F_{0}(\eta)$ and $\theta_{0}(\eta)$. These initial trials are same as given in Equations (24) and (25) for velocity and temperature, respectively.

Tables 1 and 2 show the comparative analysis among the solutions calculated by adopting variation of parameters and variational iteration methods. Furthermore, these solutions are compared with numerical solutions to check the validity of the both applied methods (VPM and VIM). It is easy to see that both analytical solutions are in magnificent agree-

\begin{tabular}{|c|c|c|c|c|c|c|}
\hline \multirow[b]{2}{*}{$\eta$} & \multicolumn{3}{|c|}{$\mathrm{Cu} /$ water } & \multicolumn{3}{|c|}{$\mathrm{Cu} /$ kerosene oil } \\
\hline & VPM & VIM & Num & VPM & VIM & Num \\
\hline 0.0 & 0 & 0 & 0 & 0 & 0 & 0 \\
\hline 0.1 & 0.084124 & 0.084124 & 0.084124 & 0.089562 & 0.089562 & 0.089562 \\
\hline 0.2 & 0.139400 & 0.139400 & 0.139400 & 0.159755 & 0.159755 & 0.159755 \\
\hline 0.3 & 0.170301 & 0.170301 & 0.170301 & 0.212844 & 0.212844 & 0.212844 \\
\hline 0 . & 0.181418 & 0.181418 & 0.181418 & 0.251099 & 0.251099 & 0.251099 \\
\hline 0.5 & 0.177425 & 0.177425 & 0.177425 & 0.276795 & 0.276795 & 0.276795 \\
\hline 0.6 & 0.163070 & 0.163070 & 0.163070 & 0.292230 & 0.292230 & 0.292230 \\
\hline 0.7 & 0.143176 & 0.143176 & 0.143 & 0.299751 & 0.299751 & 0.299751 \\
\hline 0.8 & 0.122658 & 0.122658 & 0.122658 & 0.301797 & 0.301797 & 0.301797 \\
\hline 0.5 & 0.106544 & 0.106544 & 0.106544 & 0.300949 & 0.300949 & 0.300949 \\
\hline 1.0 & 0.100000 & 0.100000 & 0.100000 & 0.299999 & 0.299999 & 0.299999 \\
\hline
\end{tabular}
ment with numerical solutions. The error for VPM and VIM is also discussed and given by Tables 3 and 4 for velocity and temperature fields, respectively.

\section{Results with Comprehensive Discussion}

This subsection is organized to investigate the behaviour of the nanoliquid motion, temperature, shear stresses, and local thermal performance rate over the region of interest. The results are captured by altering the governing parameter
TABle 1: Comparison of the solution for the velocity field.

within physical domain. These results are plotted for both sort of nanoliquids.

Figure 2 elaborates the behaviour of $\mathrm{Cu}$-kerosene oil and $\mathrm{Cu}$-water against the squeeze number $\beta$ and $\mathrm{Cu}$ fraction factor $\phi$. It is examined that the fluid motion rises due to higher $\beta$. Physically, the acceleration of the plates exerts pressure force on the fluid particles due to which these particles move rapidly in the region of interest. The rapid increment in the motion is observed in the locality of the upper Riga plate. On the other hand, higher fraction factor opposes the fluid velocity in Figure 2(b). The core reason behind this behaviour is the density of the nanofluid. By increasing the strength of $\phi$, the nanofluid becomes more thicker, and ultimately, the internal fluid forces become dominant which resists the motion.

The effects of thermal radiation, $\mathrm{Cu}$ fraction factor, and Biot number on the thermal performance of $\mathrm{Cu}$-water and $\mathrm{Cu}$-kerosene oil are furnished in Figures 3 and 4, respectively. The results plotted in Figure 3(a) ensure that the thermal performance of $\mathrm{Cu}$-water and $\mathrm{Cu}$-kerosene oil can be augmented by imposing thermal radiation effects. Physically, thermal radiation provides the heat energy to the fluid particles which leads to increment in the temperature. Similarly, the higher volumetric fraction also favors the nanofluid temperature, and these effects are pictured in Figure 3(b).

Convectively heated surface is a big source of heat transfer augmentation in the nanofluid. Therefore, the temperature alterations due to convective surface (Biot number) are decorated in Figure 4. It can be that augmentations in the heat transfer are more rapid due convectively heated surface. Near the surface, these effects are very prominent because of maximum amount of heat transfer at the surface. The particles attached to the surface transfer the energy to rest of the particles; as a result, the fluid temperature goes up.

The study of shear stresses in $\mathrm{Cu}$-water and $\mathrm{Cu}$-kerosene oil is imperative for industrial and engineering applications. Therefore, Figure 5 is designed to explore the trends of shear stresses for varying $\beta$ and $\phi$. From the results, it is 
TABLE 2: Comparison of the solutions for the temperature.

\begin{tabular}{lcccccr}
\hline$\eta$ & VPM & Cu/water & Num & VPM & \multicolumn{2}{c}{ Cu/kerosene oil } \\
\hline 0.0 & 0.137213 & 0.137213 & 0.137213 & 0.289725 & 0.289725 & 0.289725 \\
0.1 & 0.120072 & 0.120072 & 0.120072 & 0.247533 & 0.247533 \\
0.2 & 0.103522 & 0.103522 & 0.103522 & 0.207552 & 0.207552 & 0.247533 \\
0.3 & 0.087895 & 0.087895 & 0.087895 & 0.171065 & 0.171065 & 0.207552 \\
0.4 & 0.073295 & 0.073295 & 0.073295 & 0.138484 & 0.138484 & 0.171065 \\
0.5 & 0.059663 & 0.059663 & 0.059663 & 0.109623 & 0.109623 & 0.109623 \\
0.6 & 0.046852 & 0.046852 & 0.046852 & 0.083962 & 0.083962 \\
0.7 & 0.034670 & 0.034670 & 0.034670 & 0.060841 & 0.060841 & 0.083962 \\
0.8 & 0.022917 & 0.022917 & 0.022917 & 0.039574 & 0.039574 \\
0.9 & 0.011409 & 0.011409 & 0.011409 & 0.019499 & 0.019499 & 0.039574 \\
1.0 & $-1.60000 e-10$ & $-1.60000 e-10$ & 0 & $4.3 e-09$ & 0.019499 \\
\hline
\end{tabular}

TABLE 3: Error analysis between VPM and VIM for velocity.

\begin{tabular}{lcccc}
\hline$\eta$ & Error VPM & Cu-water & Error VIM & Cu-kerosene oil \\
\hline 0.0 & 0 & 0 & 0 & 0 \\
0.1 & $4.053854336 e-010$ & $3.553854294 e-010$ & $07.56226369 e-009$ & $7.522263687 e-009$ \\
0.2 & $1.140821326 e-009$ & $1.040821318 e-009$ & $2.681196706 e-008$ & $2.681196706 e-008$ \\
0.3 & $2.15307891 e-009$ & $2.053078901 e-009$ & $5.202073416 e-008$ & $5.222073418 e-008$ \\
0.4 & $3.044108965 e-009$ & $3.044108965 e-009$ & $7.806090879 e-008$ & $7.81609088 e-008$ \\
0.5 & $3.994240744 e-009$ & $3.694240719 e-009$ & $9.931656803 e-008$ & $9.961656799 e-008$ \\
0.6 & $4.081787769 e-009$ & $3.981787761 e-009$ & $1.094292154 e-007$ & $1.095292154 e-007$ \\
0.7 & $3.710801472 e-009$ & $3.410801475 e-009$ & $1.033390067 e-007$ & $1.034390067 e-007$ \\
0.8 & $2.277394731 e-009$ & $2.477394734 e-009$ & $7.577738309 e-008$ & $7.58773831 e-008$ \\
0.9 & $3.673078369 e-010$ & $3.269216842 e-011$ & $3.079392602 e-008$ & $3.139392601 e-008$ \\
1.0 & $9.999999717 e-010$ & $5.999999664 e-010$ & $1.000002303 e-010$ & $2.000002386 e-010$ \\
\hline
\end{tabular}

TABLE 4: Error analysis between VPM and VIM for temperature.

\begin{tabular}{lcccr}
\hline$\eta$ & & Cu-water & & \multicolumn{2}{c}{ Cu-kerosene oil } \\
& Error VPM & Error VIM & Error VPM & $2.269216419 e-005$ \\
0.0 & $2.917453946 e-007$ & $2.916453946 e-007$ & $2.270226419 e-005$ & $2.404027907 e-005$ \\
0.2 & $2.9763384 e-007$ & $2.9753384 e-007$ & $2.405097907 e-005$ & $2.531796947 e-005$ \\
0.3 & $3.030928012 e-007$ & $3.029928012 e-007$ & $2.532916947 e-005$ & $2.648381049 e-005$ \\
0.4 & $3.084416274 e-007$ & $3.083416274 e-007$ & $2.649541049 e-005$ & $2.750040899 e-005$ \\
0.5 & $3.128298272 e-007$ & $3.127198272 e-007$ & $2.751250899 e-005$ & $2.820559169 e-005$ \\
0.6 & $3.131458008 e-007$ & $3.130458008 e-007$ & $2.821819169 e-005$ & $2.803227177 e-005$ \\
0.7 & $3.011267026 e-007$ & $3.009967026 e-007$ & $2.804527177 e-005$ & $2.585322549 e-005$ \\
0.8 & $2.650793188 e-007$ & $2.649293188 e-007$ & $2.586572549 e-005$ & $2.042346037 e-005$ \\
0.9 & $1.982127418 e-007$ & $1.980027418 e-007$ & $2.043576037 e-005$ & $1.140035168 e-005$ \\
1.0 & $1.054550512 e-007$ & $1.050350512 e-007$ & $4.3 e-009$ & $5.4 e-009$ \\
\hline
\end{tabular}




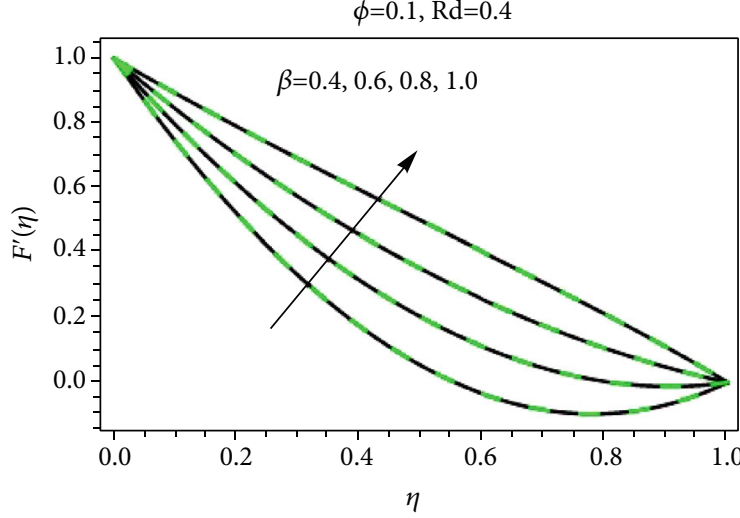

(a)

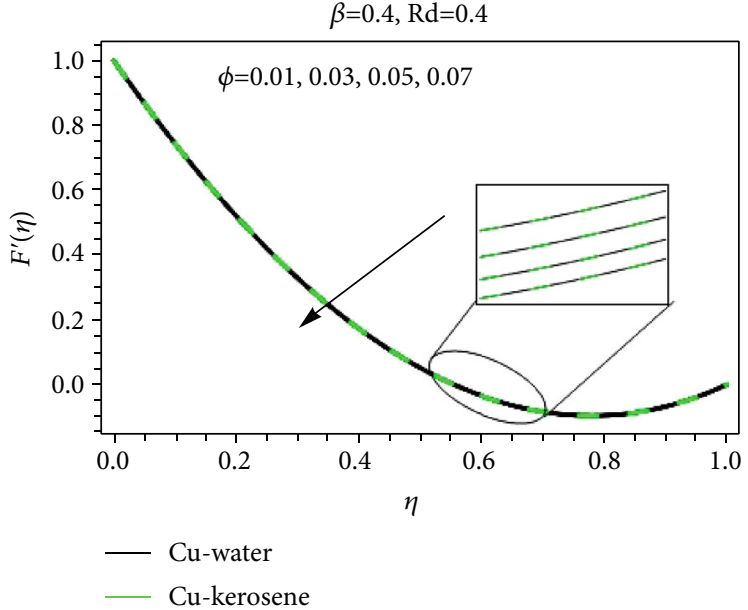

(b)

Figure 2: Impact of (a) Rd and (b) $\beta$ on $F^{\prime}(\eta)$.

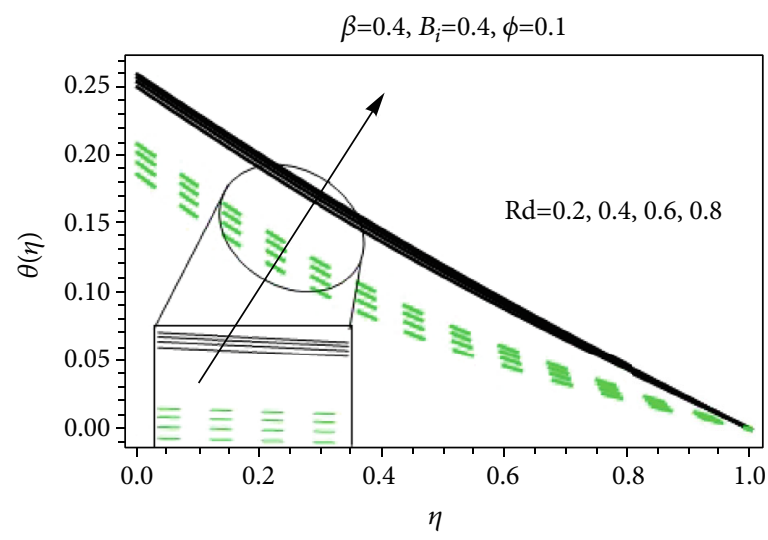

(a)

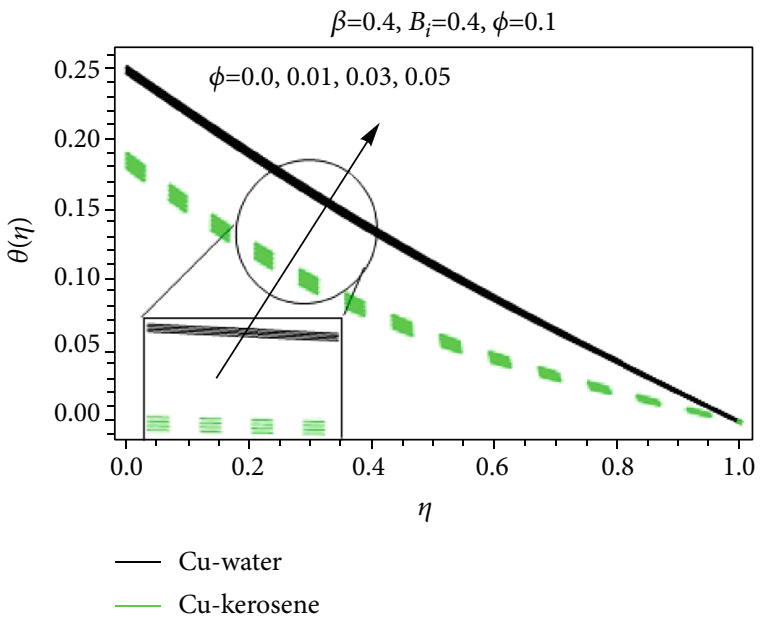

(b)

Figure 3: Impact of (a) Rd and (b) $\phi$ on $\theta(\eta)$.

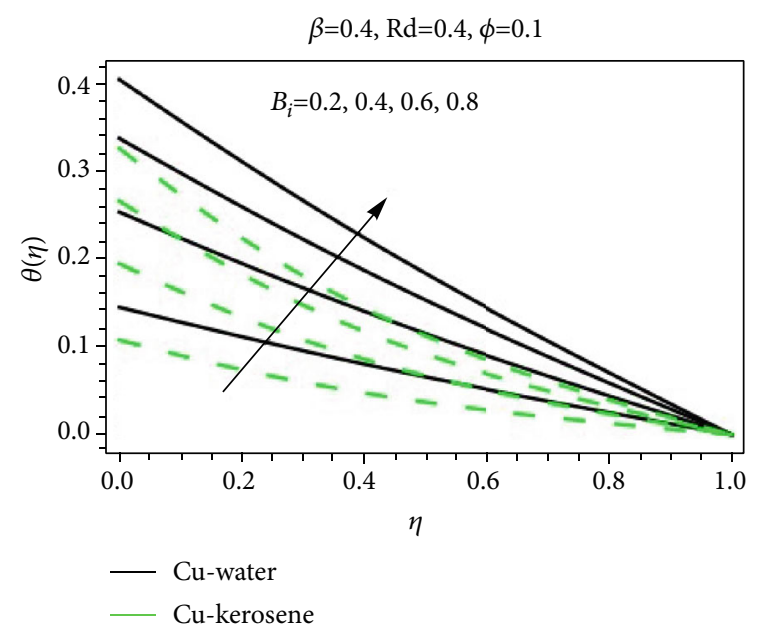

Figure 4: Impact of $B_{i}$ on $\theta(\eta)$. noteworthy that the shear stresses drop against the stronger $\beta$. For $\mathrm{Cu}$-kerosene oil, these are very rapid than $\mathrm{Cu}$-water in which thermophysical values of the base liquid and guest nanoparticles playing significant role. The slow decline in the shear stresses can be examined from Figure 5(b).

The local thermal performance in the nanofluids is of much interest due to their broad applications in the modern world. For this purpose, Figures 6-8 are decorated by considering different values of the pertinent flow parameters. It is investigated that the Biot number and thermal radiation are very beneficial for thermal performance in $\mathrm{Cu}$-water and $\mathrm{Cu}$-kerosene oil. The nanofluids gained much energy from Biot and thermal radiation parameters due to which the heat transfer rate rises. Furthermore, $\beta$ and volumetric fraction are playing important role in thermal rate of $\mathrm{Cu}$-water and $\mathrm{Cu}$-kerosene oil. Thermophysical values of the guest nanoparticles and host liquid are described in Table 5. 
$\mathrm{Rd}=0.4, B_{i}=0.4$

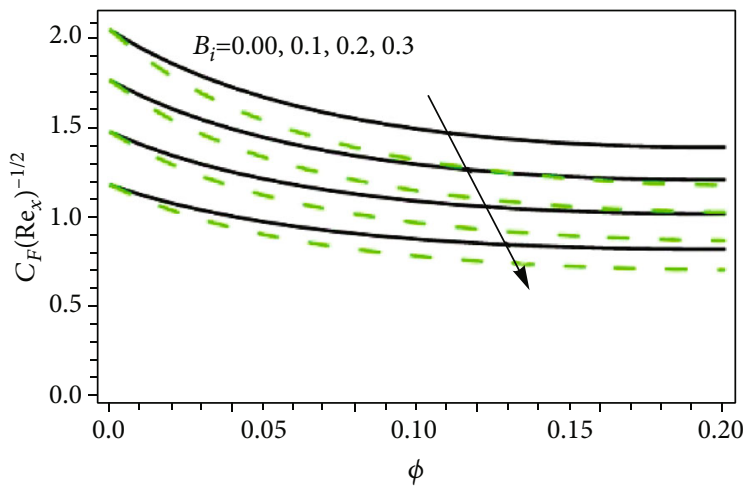

(a)

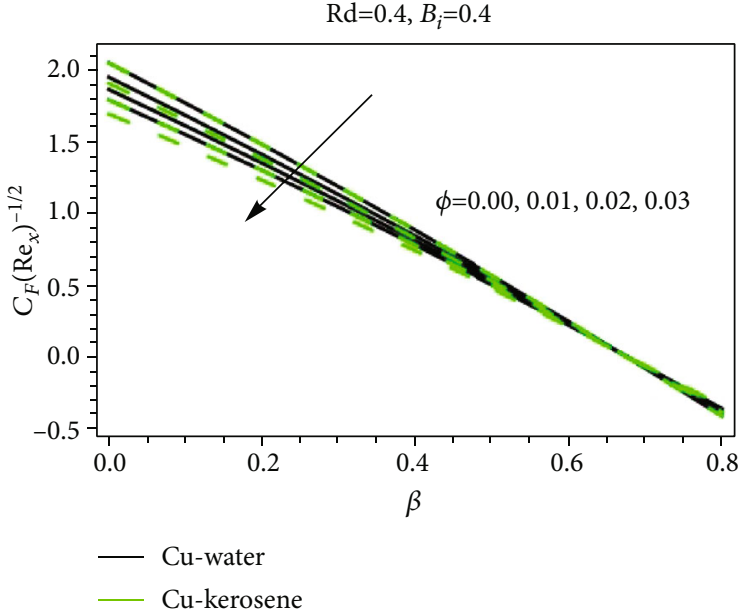

(b)

Figure 5: Impact of (a) $\beta$ and (b) $\phi$ on shear stresses.

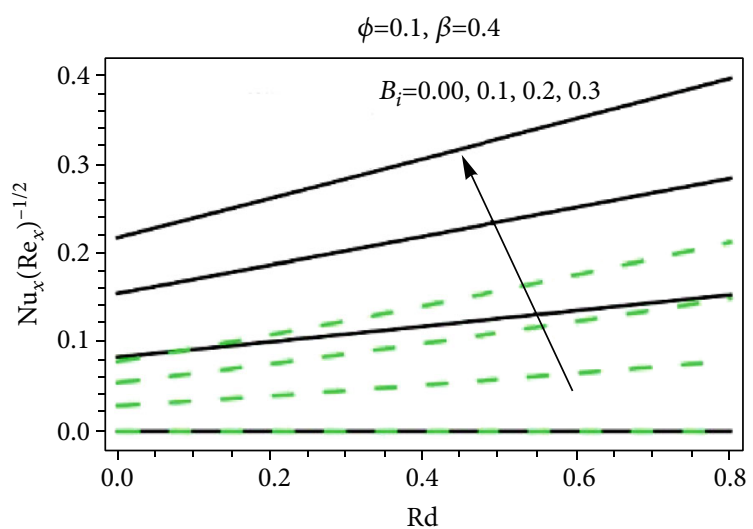

(a)

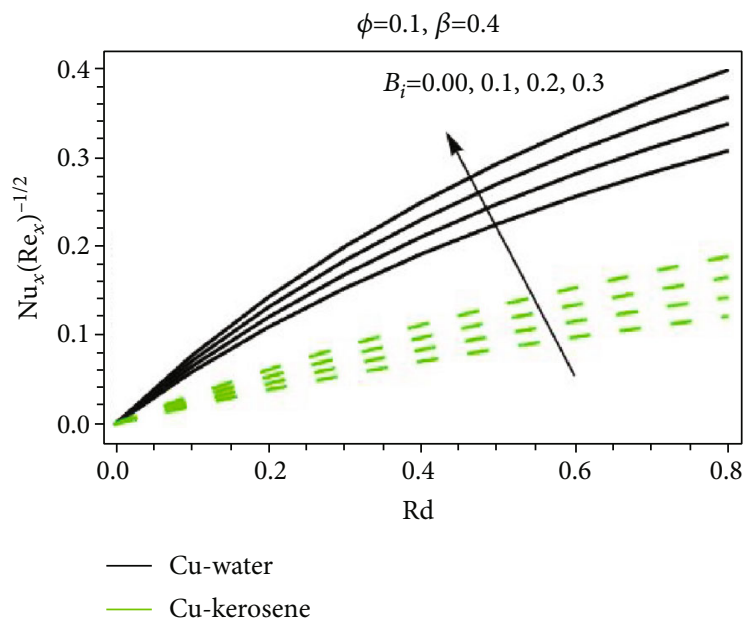

(b)

FIgURE 6: Impact of (a) $B_{i}$ and (b) Rd on Nusselt number.

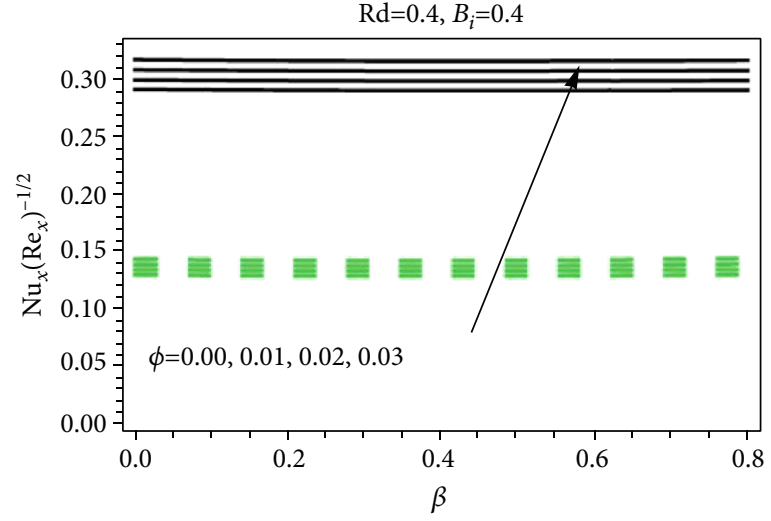

(a)

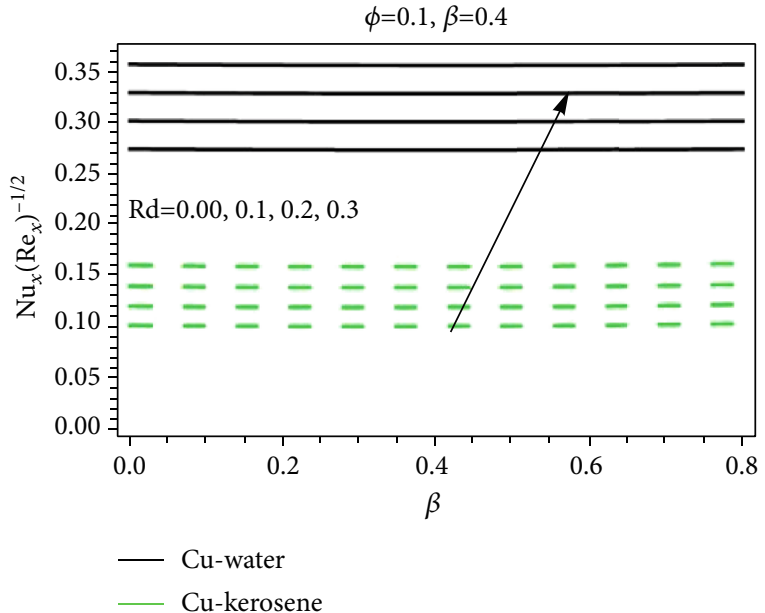

(b)

Figure 7: Impact of (a) $\phi$ and (b) Rd on Nusselt number. 


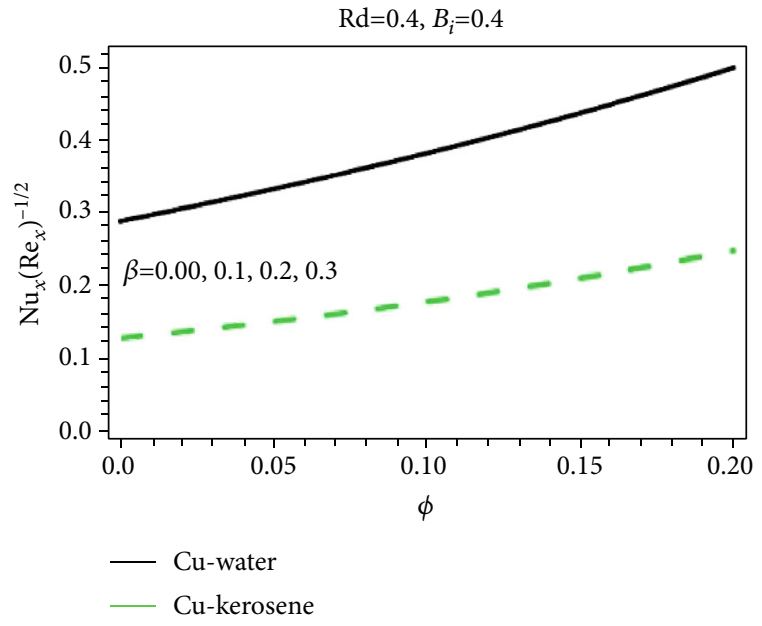

Figure 8: Impact of $\beta$ and $\phi$ on local Nusselt number.

TABLE 5: Thermo-physical values of carbon nanotubes and the regular liquids [33].

\begin{tabular}{lcccc}
\hline & $\rho\left(\mathrm{kg} / \mathrm{m}^{3}\right)$ & $C_{p}(\mathrm{~J} / \mathrm{kgK})$ & $k(\mathrm{~W} / \mathrm{mK})$ & $\operatorname{Pr}$ \\
\hline Pure water & 997.1 & 4179 & 0.613 & 6.2 \\
Kerosene oil & 783 & 2090 & 0.145 & 21 \\
Copper & 8933 & 385 & 401 & - \\
\hline
\end{tabular}

TABLE 6: Comparison with existing literature.

\begin{tabular}{lcc}
\hline$\beta$ & & $F^{\prime \prime}(1)$ \\
& Present & Hayat et al. [31] \\
\hline 0.1 & 1.69208 & 1.69635 \\
0.3 & 1.08117 & 1.08543 \\
0.5 & 0.46327 & 0.467511 \\
\hline
\end{tabular}

\section{Comparison with Existing Literature}

It is very imperative to authenticate the present analysis with previously published work. Therefore, a useful comparison is made by taking $\phi=0, Z=1.5$. The computed results are then compared with the results reported in [31]. From Table 6, it is noted that the results reported in the study are valid under aforementioned assumption which is evidence of the reliability of the study.

\section{Conclusions}

The study of nanofluids synthesized by water and kerosene oil in the presence of $\mathrm{Cu}$ guest nanoparticles is organized between two Riga plates. The similarity relations are used for the conversion of dimensional model into dimensionless form and then performed mathematical analysis. Two wellknown effective analytical techniques are adopted for the solution purpose and then decorated the results against the flow parameters over the desired region. Form the study, it is concluded as follows: (i) The velocity reduces by increasing the volumetric fraction of the nanoparticles

(ii) The temperature significantly rises for stringer thermal radiations and fraction factor $\phi$ for both $\mathrm{Cu}$ $\mathrm{H}_{2} \mathrm{O}$ and $\mathrm{Cu}$-kerosene oil nanofluids

(iii) The convectively heated plate produces extra heat to the nanofluid particles due to which it enhances rapidly

(iv) The decreasing trends in the shear stresses are rapid for $\mathrm{Cu}$-kerosene oil than $\mathrm{Cu}-\mathrm{H}_{2} \mathrm{O}$

(v) The local Nusselt number increases against thermal radiations, $B_{i}$ and $\phi$

(vi) The VPM is more effective than VIM for such nonlinear nanofluid models

\section{Nomenclature}

$(u, v)$ : Velocity components

p: $\quad$ Pressure

$T: \quad$ Temperature

$\mu_{n f}: \quad$ Nanoliquid dynamic viscosity

$k_{n f}: \quad$ Nanoliquid thermal conductivity

$\sigma^{*}: \quad$ Stefan Boltzmann constant

$k^{*}: \quad$ Mean absorption coefficient

$\phi: \quad$ Fraction factor of $\mathrm{Cu}$

$\rho_{f}: \quad$ Fluids density

$\rho_{s}: \quad$ Cu density

$\Psi: \quad$ Stream function

$\eta$ : $\quad$ Self-similar variable

$n f: \quad$ Stands for nanoliquid

$F(\eta)$ : Dimensionless velocity

$\theta$ : Dimensionless temperature

Rd: Radiation number

Pr: Prandtl number

$B_{i}$ : $\quad$ Biot number.

\section{Data Availability}

The study based on numerical technique and no data is used in findings of the study.

\section{Conflicts of Interest}

The authors declare no financial/competing interest regarding this work.

\section{Authors' Contributions}

A. and W.A. wrote the original draft. Problem formulation was done by A.H.A., I.K., and A. M.A., A., I.K., and A.H.A. contributed to the results and discussion. Validity of the study was done by A. and W.A. Software was done by M.A. and I.K. Language correction was done by A., A.H.A., and M.A. 


\section{References}

[1] S. Choi, "Enhancing thermal conductivity of fluids with nanoparticles in developments and applications of non-Newtonians flows," ASME, vol. 66, pp. 99-105, 1995.

[2] S. T. Mohyud-Din, U. Khan, N. Ahmed, and B. Bin-Mohsin, "Heat and mass transfer analysis for MHD flow of nanofluid inconvergent/divergent channels with stretchable walls using Buongiorno's model," Neural Computing and Applications, vol. 28, no. 12, pp. 4079-4092, 2017.

[3] U. Khan, N. Ahmed, S. T. Mohyud-Din, and B. Bin-Mohsin, "Nonlinear radiation effects on MHD flow of nanofluid over a nonlinearly stretching/shrinking wedge," Neural Computing and Applications, vol. 28, no. 8, pp. 2041-2050, 2017.

[4] M. Sheikholeslami, "CVFEM for magnetic nanofluid convective heat transfer in a porous curved enclosure," The European Physical Journal Plus, vol. 131, no. 11, pp. 131-413, 2016.

[5] M. Sheikholeslami, "Influence of Lorentz forces on nanofluid flow in a porous cylinder considering Darcy model," Journal of Molecular Liquids, vol. 225, pp. 903-912, 2017.

[6] K. U. Adnan, U. Khan, N. Ahmed, and S. T. Mohyud-Din, "Thermo-diffusion and diffusion-thermo effects on flow of second grade fluid between two inclined plane walls," Journal of Molecular Liquids, vol. 224, pp. 1074-1082, 2016.

[7] M. Sheikholeslami and H. B. Rokni, "Nanofluid two phase model analysis in existence of induced magnetic field," International Journal of Heat and Mass Transfer, vol. 107, pp. 288-299, 2017.

[8] M. S. Kandelous, "KKL correlation for simulation of nanofluid flow and heat transfer in a permeable channel," Physics Letters A, vol. 378, no. 45, pp. 3331-3339, 2014.

[9] A. M. Adnan, M. Asadullah, U. Khan, N. Ahmed, and S. T. Mohyud-Din, "Analytical and numerical investigation of thermal radiation effects on flow of viscous incompressible fluid with stretchable convergent/divergent channels," Journal of Molecular Liquids, vol. 224, pp. 768-775, 2016.

[10] A. Saeed, P. Kumam, S. Nasir, T. Gul, and W. Kumam, "Nonlinear convective flow of the thin film nanofluid over an inclined stretching surface," Scientific Reports, vol. 11, no. 1, article 97576, 2021.

[11] A. Gul, I. Khan, and S. Shafie, "Energy transfer in mixed convection MHD flow of nanofluid containing different shapes of nanoparticles in a channel filled with saturated porous medium naniscale," Nanoscale Research Letters, vol. 10, 2015.

[12] A. Gul, I. Khan, and S. Shafie, "Radiation and heat generation effects in MHD mixed convection flow of nanofluids," Thermal Science, vol. 22, 2018.

[13] N. A. Mohd Zin, I. Khan, and S. Shafie, "The impact silver nanoparticles on MHD free convection flow of Jeffrey fluid over an oscillating vertical plate embedded in a porous medium," Journal of Molecular Liquids, vol. 222, pp. 138150, 2016.

[14] A. Khalid, I. Khan, and S. Shafie, "Heat transfer in ferrofluid with cylindrical shape nanoparticles past a vertical plate with ramped wall temperature embedded in a porous medium," Journal of Molecular Liquids, vol. 221, pp. 1175-1183, 2016.

[15] M. J. Stefan, "Versuch uber die scheinbare Adhasion, sitzungsber, Abt II Osterr," Annalen der Physik, vol. 69, pp. 316-318, 1874.

[16] M. M. Rashidi, H. Shahmohamadi, and S. Dinarvand, "Analytic Approximate Solutions for Unsteady Two-Dimensional and Axisymmetric Squeezing Flows between Parallel Plates," Mathematical Problems in Engineering, vol. 2008, Article ID 935095, 13 pages, 2008.

[17] U. Khan, N. Ahmed, M. Asadullah, and S. Tauseef Mohyuddin, "Effects of viscous dissipation and slip velocity on twodimensional and axisymmetric squeezing flow of $\mathrm{Cu}$-water and Cu-kerosene nanofluids," Propulsion and Power Research, vol. 4, no. 1, pp. 40-49, 2015.

[18] A. Gailitis and O. Lielausis, "On a possibility to reduce the hydrodynamic resistance of a plate in an electrolyte," Applied Magnetohydrodynamics, vol. 12, pp. 143-146, 1961.

[19] M. D. Shamshuddin, S. R. Mishra, O. Anwar Beg, and A. Kadir, "Adomian decomposition method simulation of von Kármán swrling bioconvection nanofluid flow," Journal of Central South University, vol. 26, no. 10, pp. 2797-2813, 2019.

[20] M. D. Shamshuddin, S. R. Mishra, O. A. Bég, and A. Kadir, "Viscous dissipation and joule heating effects in non-Fourier MHD squeezing flow, heat and mass transfer between Riga plates with thermal radiation: variational parameter method solutions," Arabian Journal for Science and Engineering, vol. 44, no. 9, pp. 8053-8066, 2019.

[21] Y. X. Li, T. Muhammad, M. Bilal, M. A. Khan, A. Ahmadian, and B. A. Pansera, "Fractional simulation for DarcyForchheimer hybrid nanoliquid flow with partial slip over a spinning disk," Alexandria Engineering Journal, vol. 60, no. 5, pp. 4787-4796, 2021.

[22] M. Bilal, A. Saeed, T. Gul, I. Ali, W. Kumam, and P. Kumam, "Numerical approximation of microorganisms hybrid nanofluid flow induced by a wavy fluctuating spinning disc," Coatings, vol. 11, no. 9, p. 1032, 2021.

[23] A. R. Ehman, Z. Salleh, and T. Gul, "Heat transfer of thin film flow over an unsteady stretching sheet with dynamic viscosity," Journal of Advanced Research in Fluid Mechanics and Thermal Sciences, vol. 81, no. 2, pp. 67-81, 2021.

[24] H. R. Kataria and A. S. Mittal, "Velocity, mass and temperature analysis of gravity-driven convection nanofluid flow past an oscillating vertical plate in the presence of magnetic field in a porous medium," Applied Thermal Engineering, vol. 110, pp. 864-874, 2017.

[25] H. R. Kataria and A. S. Mittal, "Mathematical model for velocity and temperature of gravity-driven convective optically thick nanofluid flow past an oscillating vertical plate in presence of magnetic field and radiation," Journal of the Nigerian Mathematical Society, vol. 34, no. 3, pp. 303-317, 2015.

[26] M. Sheikholeslami, H. R. Kataria, and A. Mittal, "Effect of thermal diffusion and heat-generation on MHD nanofluid flow past an oscillating vertical plate through porous medium," Journal of Molecular Liquids, vol. 257, pp. 12-25, 2018.

[27] N. Ahmed, Adnan, U. Khan, and S. T. Mohyud-Din, "Influence of shape factor on flow of magneto-nanofluid squeezed between parallel disks," Alexandria Engineering Journal, vol. 57, no. 3, pp. 1893-1903, 2018.

[28] M. Turkyilmazoglu, "Nanoliquid film flow due to a moving substrate and heat transfer," European Physical Journal Plus, vol. 135, no. 10, 2020.

[29] A. A. Siddiqui and M. Turkyilmazoglu, "A new theoretical approach of wall transpiration in the cavity flow of the ferrofluids," Micromechanics, vol. 10, no. 6, p. 373, 2019.

[30] J. A. Khan, M. Mustafa, T. Hayat, M. Turkyilmazoglu, and A. Alsaedi, "Numerical study of nanofluid flow and heat 
transfer over a rotating disk using Buongiorno's model," International Journal of Numerical Methods for Heat \& Fluid Flow, vol. 27, no. 1, pp. 221-234, 2017.

[31] T. Hayat, M. Khan, M. Imtiaz, and A. Alsaedi, "Squeezing flow past a Riga plate with chemical reaction and convective conditions," Journal of Molecular Liquids, vol. 96, 2017.

[32] M. Sheikholeslami, T. Hayat, and A. Alsaedi, "MHD free convection of $\mathrm{Al}_{2} \mathrm{O}_{3}$-water nanofluid considering thermal radiation: A numerical study," International Journal of Heat and Mass Transfer, vol. 96, pp. 513-524, 2016.

[33] U. Khan, N. Ahmed, and S. T. Mohyud-Din, "Numerical investigation for three dimensional squeezing flow of nanofluid in a rotating channel with lower stretching wall suspended by carbon nanotubes," Applied Thermal Engineering, vol. 113, pp. 1107-1117, 2017. 